\title{
Emerging alphaviruses in the Americas: Chikungunya and Mayaro
}

\author{
Mario Luis Garcia de Figueiredo ${ }^{[1],[2]}$ and Luiz Tadeu Moraes Figueiredo ${ }^{[2]}$
}

[1]. Instituto Evandro Chagas, Belém, PA. [2]. Centro de Pesquisa em Virologia, Faculdade de Medicina de Ribeirão Preto, Universidade de São Paulo, Ribeirão Preto, SP.

\begin{abstract}
Chikungunya virus (CHIKV) and Mayaro virus (MAYV) are emergent arthropod-borne viruses that produce outbreaks of acute febrile illness with arthropathy. Despite their different continental origins, CHIKV and MAYV are closely related and are components of the Semliki Forest Complex of the Alphavirus (Togaviridae). MAYV and, more recently, CHIKV, which are both transmitted by Aedes mosquitoes, have resulted in severe public health problems in the Americas, including Brazil. In this review, we present aspects of the pathogenesis, clinical presentation and treatment of febrile illnesses produced by CHIKV and MAYV. We also discuss the epidemiological aspects and effects related to the prophylaxis of infections by both viruses.
\end{abstract}

Keywords: Emerging alphaviruses. Chikungunya fever. Mayaro fever.

\section{INTRODUCTION}

The spread of ribonucleic acid (RNA) viruses has been facilitated by environmental degradation and population growth. In large urban agglomerations, there are increasing opportunities for viral contagion. Viruses spread rapidly across large distances because of human travel in fast vehicles, such as airplanes. Thus, viruses can travel in infected passengers, especially individuals in the incubation period of disease. Migrations to urban areas and the uncontrolled growth of cities can also increase the spread of pathogens. Thus, these pathogens transition from a health problem in a restricted area to a worldwide problem ${ }^{1}$. Some RNA viruses have emerged and are currently producing pandemics. Examples of these viruses include dengue (Flavivirus) and Chikungunya (Alphavirus), both of which are arthropod-borne viruses.

Emerging viruses typically originate in resource-poor settings and without a health surveillance structure. These viruses are better studied only after they reach distant places from their origins. The emergence of an arbovirus (arthropodborne viruses) is related to ecological, environmental or climate changes that affect its ecological niche. The geographical displacement of arboviruses is also related to the spread of animal reservoirs and/or their arthropod vectors (mosquitoes and ticks). Migrations that cause the invasion of arbovirus

Address to: Dr. Mario Luis Garcia de Figueiredo. Centro de Pesquisa em Virologia/FMRP/USP. Av. Bandeirantes 3900, Monte Alegre, 14049-900 Ribeirão Preto, SP, Brasil.

Phone: 55 16 3602-4589; Fax: 5516 3602-3376

e-mail: marioluisgf@yhoo.com.br

Received 14 October 2014

Accepted 19 November 2014 ecological niches could lead to virus infections and emergencies. Therefore, zoonotic viruses that adapt to urban cycles, which become transmissible by anthropophilic mosquitoes, tend to be successful as emerging pathogens ${ }^{3}$. This phenomenon is the case for Chikungunya virus (CHIKV), which is transmitted by Aedes aegypti and Aedes albopictus. Mayaro virus (MAYV) can infect humans that invade its zoonotic habitat, including primate reservoirs. In addition, CHIKV and MAYV can be vertically transmitted via a transovarian route when the virus is transmitted from the adult mosquito to its progeny ${ }^{4,5}$.

CHIKV and MAYV are able to mutate and/or adapt to new zoonotic cycles and thus acquire a higher potential for emergency. These arboviruses may emerge as a result of environmental degradation and socio-economic disturbances ${ }^{6}$. Climate changes that interfere with human activities influence the emergence of these pathogens and enhance their geographical spread.

\section{GENOME ORGANIZATION AND VIRAL REPLICATION}

CHIKV and MAYV are members of the family Togaviridae, genus Alphavirus. These viruses are $70 \mathrm{~nm}$ in diameter, have an enveloped icosahedral capsid and have a positive polarity singlestranded RNA (the genomic RNA itself is the messenger RNA). The genome of CHIKV has approximately 11,800 nucleotides, including a $5^{\prime}$ cap and a $3^{\prime}$ poly-A tail. The $5^{\prime}$ non-translated region (NTR) is composed of 76 nucleotides, and the $3^{\prime}$ NTR has 526 nucleotides; there are 68 non-translated nucleotides at the junction region of the 2 viral open reading frames (ORFs). The sequences at the $5^{\prime}$ and $3^{\prime}$ ends, as well as in the intergenic region, have conserved repeated sequence elements that play important roles in the regulation of viral RNA synthesis ${ }^{7}$. The viral genome codes for $2 \mathrm{ORFs}$. The non-structural proteins are encoded at the $5^{\prime}$ two-thirds of the genome in an ORF of 7,425 
nucleotides initiated by a ATG start codon (at position 77 \pm 79 ) and terminated at a stop codon TAG (at position 7,499 $\pm 7,501$ ). The polyprotein translated from this ORF has 2,474 amino acids, from which 4 non-structural proteins (nsP1, nsP2, nsP3 and nsP4) are formed by proteolytic cleavage. These non-structural proteins are necessary for the transcription and replication of viral RNA. The structural proteins are encoded by 4,327 nucleotides located at a subgenomic messenger ribonucleic acid (mRNA) that is colinear with the $3^{\prime}$ one-third of the genome, which starts at position 7,498 and excludes the poly (A) tail. Another ORF, with 3,735 nucleotides, is initiated by a start codon at position 7,567 7,569 and terminated by a stop codon at position $11,299 \pm 11,301$. This ORF encodes for a polyprotein of 1,244 amino acids, from which 5 structural proteins (capsid - CP, E3, $\mathrm{E} 2,6 \mathrm{~K}$ and E1) are formed after proteolytic cleavage ${ }^{8,9}$.

The envelope glycoproteins E2 and E1 are transmembrane type I and appear as triplicate membrane-anchored heterodimers. These glycoproteins correspond to the envelope spikes that coat the virus surface. E2 and E1 are responsible for receptor recognition and entry of the virus into target cells by membrane fusion. Both transmembrane glycoproteins are antigenic, and the E2 antigens are capable of inducing neutralizing antibodies, which are essential to efficient immune responses by the infected $\operatorname{host}^{10}$. The immunogenicity of CHIKV E2 has aroused great interest as a target for new methods of infection diagnosis, as well as a component of future vaccines 8 .

Despite their different continental origins, CHIKV and MAYV are closely related and are components of the Semliki Forest Complex of the Alphavirus (Togaviridae). Alphavirus mutations that confer a better fitness to anthropophilic vectors may become able to spread worldwide. These mutations can be caused by viral polymerases that incorporate high rates of errors into copied RNAs. Thus, progenies of an RNA virus with small mutations are organized into complex and dynamic communities referred to as quasispecies ${ }^{6,11}$. Certain quasispecies may include viruses that are best suited to certain moments and selective conditions. Changes in the nucleotide sequence of a viral genome can alter the amino acid sequence of proteins and, in most cases, induce a defective virus that cannot replicate. However, a virus that has developed a better infectivity may eventually arise in nature. Additionally, the replication of RNA viruses is only possible under unstable borderline ranges of errors, and some mutants maintain the informational content of the species and ability to escape the host immune system. CHIKV is a successful example of a mutant virus able to produce pandemics ${ }^{7}$. A mutation in the CHIKV genome led to the origin of viruses with a better transmission capacity by Aedes albopictus. This mutation resulted in the spread of CHIKV into the Indian Ocean and Asia, which produced a large epidemic. In contrast, MAYV is an arbovirus transmitted by Haemagogus mosquitoes that feed on primates that live in tree canopies ${ }^{12}$. This virus causes sporadic cases or outbreaks of febrile illness in the Amazon region, including large cities, such as Manaus ${ }^{13}$. A MAYV mutant could adapt to anthropophilic mosquito vectors and spread toward northeastern and southeastern Brazil, as well as neighboring countries, which would result in an even more serious public health problem.

\section{CHIKUNGUNYA VIRUS}

Chikungunya virus is a zoonotic arbovirus that was first isolated from an African primate in $1952^{14}$. CHIKV, similar to dengue virus, is transmitted to humans in urban cycles by Aedes aegypti and Aedes albopictus vectors.

After inoculation by the Aedes bite, CHIKV typically reaches the subcutaneous tissue and is phagocytosed by dendritic cells that migrate to lymph nodes to present the virus antigens to the immune system. An innate immune response is the first barrier against CHIKV, which is predominately based on an interferon that limits the spread of the virus ${ }^{15}$. The virus multiplies in monocyte/macrophage cells and interacts with these cells and other blood leukocytes, which induces the production of interferon alfa (IFN- $\alpha$ and other cytokines. Virusinfected monocyte/macrophages reach blood vessels and spread toward target organs, such as the liver, muscle tissues, joints and the brain. A vigorous immune response limits the disease produced by CHIKV and confers protection from re-infection. Thus, the migration of monocyte/macrophages into the synovial tissues likely contributes to the inflammation of joints and may explain the persistence of arthritis despite the lack of viremia ${ }^{16}$. In addition, the persistence of CHIKV in the joints of monkeys has been associated with chronic arthropathy. The virus can be detected in joint tissues for up to 90 days, leading to local inflammation ${ }^{17}$. Whether the immune response, the persistence of CHIKV in joints, or both factors could lead to this chronic disease remains unclear. Regulatory mechanisms that impair a vigorous joint inflammatory response may prevent chronic disease. High levels of Th1-type cytokines in the blood of patients could also be related to chronic arthropathy ${ }^{18}$. Thus, the chronic disease produced by CHIKV is likely induced by deregulated inflammation during the acute phase of disease and/ or convalescence. This deregulation is deleterious and could cause the inflammatory process to continue for many months after the acute disease ${ }^{19}$. In addition, CHIKV mutations, such as the mutations that occurred with the East, Central and South African genotypes of CHIKV, could induce more virulent pathogens that are able to interfere with the innate defense system by damaging the activation of the IFN response ${ }^{20}$. However, the knowledge regarding the pathogenesis of the disease produced by CHIKV remains limited, and further studies are necessary to better understand its mechanisms.

Based on clinical studies with patients from Asia and the islands of the Indian Ocean, Chikungunya fever typically manifests after an incubation period of 2-4 days after the mosquito bite. Viremia persists for up to 5 days after the onset of symptoms, and the disease includes a fever $(92 \%$ of the cases) typically associated with arthralgia (87\%), back pain $(67 \%)$ and headache $(62 \%)$. The fever occurs with a sudden onset, which reaches $39-40^{\circ} \mathrm{C}$, with chills; it typically lasts for 24-48 hours. Joint pain is typically more intense in the morning, and the majority of patients may have a migratory polyarthritis, even with joint effusion. The ankles, wrists and small joints of the hands are the most affected; however, larger joints, such as the knee, shoulder and spine, may also be involved. The patients with occupations that require an excessive use 
of small joints tend to be the most affected. Following the fever, with 2-5 days of illness, a non-pruritic maculopapular rash may arise on the face, limbs and trunk, which lasts up to 10 days. Neurological manifestations include febrile seizures, meningitis and encephalitis. Ocular manifestations, including neuroretinitis and uveitis, are common. The fever, arthritis and rash may reappear after a period of apparent remission. In some cases, debilitating arthritis can occur. Shock or death are rare in CHIKV infections. Chikungunya fever enables a differential diagnosis from dengue fever. However, for Chikungunya fever, the convalescence requires a longer resting period than dengue fever and leads to longer periods without working ${ }^{21}$. A study that analyzed the articular disease induced by CHIKV demonstrated complete resolution in $87.9 \%$ of cases, whereas $3.7 \%$ had episodic pain and joint stiffness, $2.8 \%$ had persistent stiffness without pain and $5.6 \%$ had persistent painful joint motion that represented an important constraint for the patient ${ }^{22}$. The involvement of muscles and the Achilles tendon have also been observed. Despite the low mortality, Chikungunya fever leads to important economic and social impacts that are higher than the impacts produced by other viruses.

Based on small differences in E1, E2 and nsP1 viral genes, 3 genotypes of CHIKV have been reported to date: the Asian, the West African, and the East, Central and South African $(\text { ECSA })^{23,24}$. Starting in 2005, after a mutation of the aminoacid alanine for valine at position 226 of the E1 glycoprotein, CHYKV ECSA became more adapted to transmission by Aedes albopictus but maintained the same tropism for Aedes aegypti ${ }^{25}$. ECSA CHIKV spread across the Indian Ocean, which caused major epidemics of acute febrile illness with arthralgia, meningoencephalitis and abortions in the islands of Reunion, Madagascar, Mauritius, Seychelles and Mayotte. In 2006, CHIKV arrived in India and produced an epidemic in the State of Andhra Pradesh ${ }^{26}$. In 2007, the virus caused more than 1 million infections in India, with 1,200 deaths. In 2007, CHIKV was reported in Italy near the Adriatic Sea, which produced 220 cases and 1 death, reflecting an ability to become widespread. The Italian outbreak was likely initiated by an imported case that came to a town infested by Aedes albopictus ${ }^{26}$. Imported cases of CHIKV have been reported in Europe and the United States. In South America, French Guiana reported 30 cases of Chikungunya fever imported from India in $2006^{27}$.

CHIKV of the Asian genotype was introduced in the Americas through the Caribbean, and the first autochthonous cases were reported in Saint Martin and the French West Indies in December 2013. In March 2014, Chikungunya fever was recorded in nine Caribbean islands, with more than 15,000 suspected cases ${ }^{28,29}$. CHIKV was also detected in blood donors, which raised concern and directly threatened the safety of blood products that could transmit the virus through transfusion ${ }^{30}$. In August 2014, the epidemic reached Central America (Panama, Nicaragua and El Salvador) and South America. More than 200 suspected cases were reported in Venezuela, and over 1,000 suspected cases were reported in Colombia ${ }^{31}$.

In Brazil, the Ministry of Health has reported imported cases of Chikungunya fever since $2010^{32}$. By 2014, tens of imported cases were reported in travelers from Haiti, the Dominican Republic and French Guiana. The first report of the autochthonous transmission of CHIKV in Brazil was on September 12, 2014, when the Ministry of Health confirmed two indigenous cases of Chikungunya fever in the State of Amapá. In September 2014, an outbreak by another genotype of CHIKV (the Asian genotype) arose in Feira de Santana City, State of Bahia, with hundreds of suspected cases. The situation regarding CHIKV in Bahia has rapidly evolved; in the first week of October 2014, 722 suspected cases of Chikungunya fever were reported in 10 cities, and cases were also reported in State of Minas Geraes ${ }^{32}$ (Figure 1).

Considering that vaccines or antiviral drugs to CHIKV are not available and that Brazilian cities are infested by Aedes aegypti and Aedes Albopictus, the epidemiological control of CHIKV epidemics should be based only on the traditional fight against vector mosquitoes. Therefore, recognizing the inefficient control of dengue, which is transmitted by the same mosquito, Chikungunya epidemics will likely spread rapidly throughout Brazil, whose inhabitants are completely susceptible to the virus. A recent Brazilian experimental study that analyzed the transmission of CHIKV by mosquitoes demonstrated that 35 strains of Ae. aegypti and Ae. Albopictus were capable of transmission with great efficiency, including all 3 CHIKV genotypes ${ }^{29}$. Therefore, this study reinforces the idea that CHIKV will spread in the Americas and, in particular, Brazil.

\section{MAYARO VIRUS}

MAYV was originally isolated in Trinidad in 1954 from the serum of febrile patients $\mathrm{s}^{33}$. Both genotypes of MAYV (D and L) exhibit a close phylogenetic relationship with the Una virus. The D genotype of MAYV has been reported in the Central Plateau and the Amazon region of Brazil, as well as in Peru, Bolivia, Venezuela, Colombia, Argentina and Trinidad. The L genotype appears to occur only in the Amazon region of the Brazilian State of Pará. MAYV has produced outbreaks of acute febrile illness in these places ${ }^{34}$. Typically, MAYV affects individuals who work or reside in contact with the natural environment. However, outbreaks in large cities have also been reported ${ }^{13}$. The disease caused by Mayaro lasts for 3-5 days and includes fever, headache, myalgia, rash and arthralgia of large joints and, occasionally, arthritis. The convalescence of this disease may require several weeks ${ }^{12,13}$. The physiopathology of Mayaro fever has not been studied.

MAYV is maintained in nature as a zoonosis of primates, who become infected by the bite of Haemagogus mosquitoes that live in treetops. This maintenance cycle is similar to that of sylvatic yellow fever (YFV), and the places that suffer MAYV outbreaks could also have outbreaks of YFV, which occurred in the City of Santa Cruz de la Sierra, Bolivia, in the 1980s $\mathrm{s}^{12,35}$. A study of 335 blood samples of indigenous communities in the Rio Negro region, Brazil, indicated that $41.5 \%$ of those tested had antibodies against MAYV, which demonstrates that it is a common infection in this region ${ }^{5}$. Cases of Mayaro fever have also occurred in Manaus, which is a large city and the capital of the State of Amazonas, in 2007-2008. In this city, immunoglobulin M (IgM) antibodies to MAYV were detected 


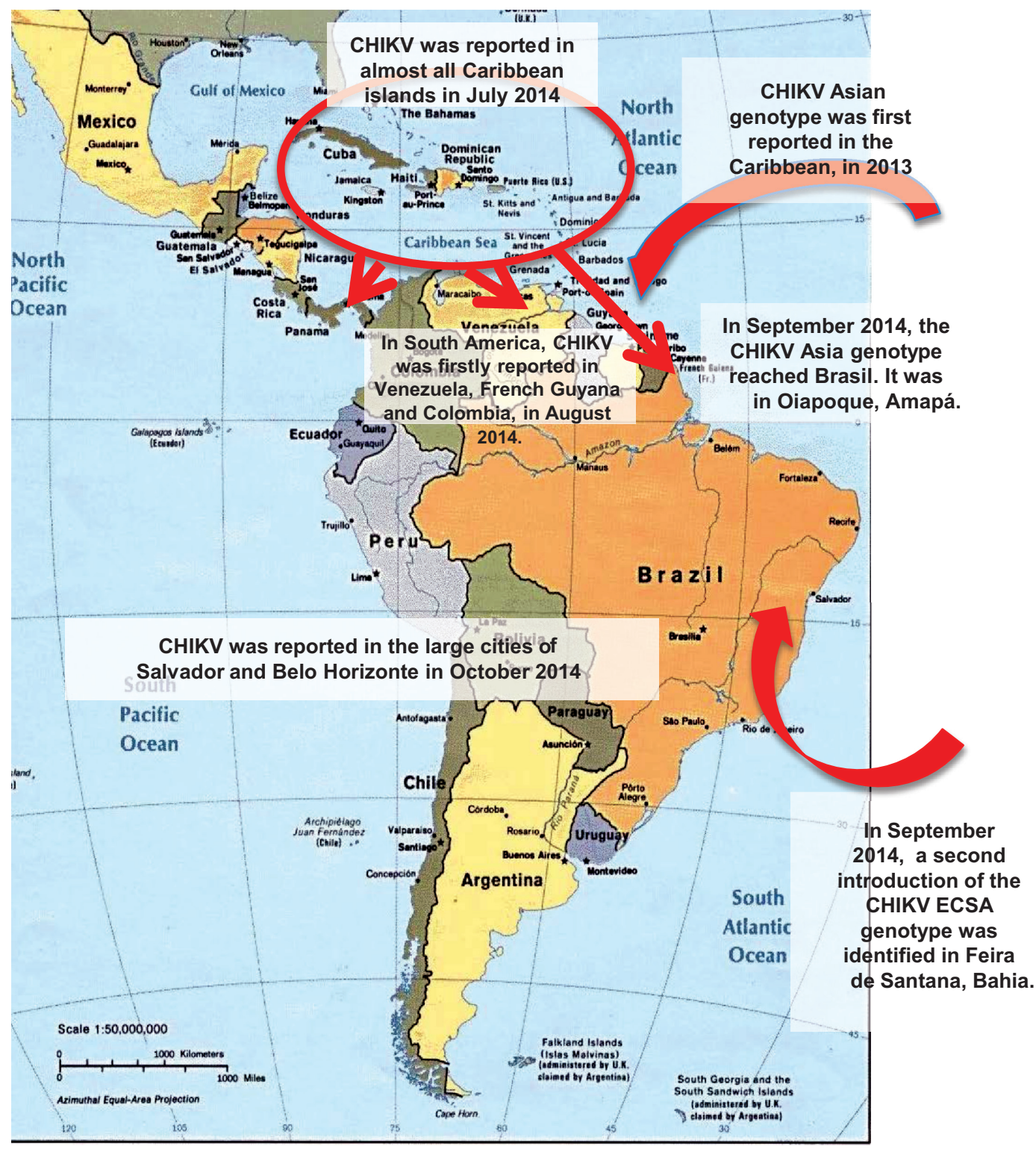

FIGURE 1 - Emergence of Chikungunya virus in the Americas. CHIKV: Chikungunya; ECSA: East, Central and South African.

by an enzyme immunoassay in the sera of 33 patients aged 6 to 65 years. The MAYV genome was identified in one of these sera. The patients had fever, headache, arthralgia, eye pain and rash. It is possible that these patients were the tip of an iceberg that reflected many undiagnosed cases ${ }^{13}$. Evidence of MAYV migration to other parts of Brazil has also been reported. In 2000, 3 patients who had Mayaro fever and most likely acquired it in Camapuã City, located in the Center-West of the country, traveled to the City of São Paulo, where they were diagnosed ${ }^{36}$. In addition, antibodies to MAYV have been observed in 2 birds captured in the State of Rio Grande do Sul in the south of Brazil and far away from the Amazon region (Araujo AA, Ministry of Health: personal communication, 2005). These data indicate a risk of the introduction of MAYV to other regions of Brazil. Thus, MAYV is an emerging virus that affects human populations, even individuals who live in the large cities of the
Amazon region. This virus could spread to other regions of Brazil, as well as other tropical American countries, because it is introduced by sick travelers or migratory birds. MAYV and CHIKV could also be transmitted by Aedes aegypti. Therefore, it is possible to imagine a future scenario in which MAYV, after adaptation to an urban cycle, causes major epidemics ${ }^{2,36}$.

\section{LABORATORY DIAGNOSIS}

Rapid, sensitive and reliable laboratory methods should be used for the diagnosis of infections by both CHIKV and MAYV. These techniques should be used to provide a better management of patients, as well as for the detection and control of outbreaks. Molecular assays are rapid and sensitive tools for the diagnosis of infections by these viruses in the early viremic stages of illness. Conventional real time polymerase-chain reaction (RT-PCR) have been used. These techniques use generic primers 
for Alphavirus or virus-specific primers that amplify the genes $\mathrm{nsP} 1$, nsP2 or $\mathrm{E} 1^{13,20,37,38}$. Viral isolation can be performed on the first or second day of the disease from the serum of viremic patients inoculated into C6/36 insect cells or Vero-E6 monkey cells. Virus isolation can also be performed by the intracerebral inoculation of baby mice ${ }^{39,40}$. Early antibodies can be detected in patient sera as early as after 5 days after the onset of symptoms. These antibodies neutralize the virus, thus preventing its isolation. The isolation of CHIKV and MAYV requires at least 3 days and is important for investigating the epidemiology and pathogenesis of these viruses. The isolation of CHIKV and MAYV also allows for their molecular characterization.

The serologic response to infections by CHIKV and MAYV can be detected by enzyme-immune assays (EIAs), an indirect immunofluorescence assay (IFA), a hemoagglutination inhibition (HI) test and a neutralization test (NT). Enzyme immunoassay is a rapid and sensitive technique for the detection of specific antibodies and can discriminate between immunoglobulin $\mathrm{G}$ (IgG) and immunoglobulin M (IgM). The IgM to CHIKV is typically detectable 3 days after the onset of symptoms and persists up to 3 months. IgG antibodies to CHIKV and MAYV appear after IgM antibodies and persist for years. Various EIA techniques have been prepared in-house for the diagnosis of these viral infections. Some techniques use virus-infected cells, the whole virus or recombinant viral envelope or nonstructural proteins as the antigens $s^{5,13,20,33,41}$. Commercial serological assays, including fast methods, are also available; however, their sensitivities for antibody detection before day 5 after the onset of symptoms is dependent on the virus strain or the source of the antigen used for the assay. Assays based on recombinant antigens might be more specific regarding virus genotype and mutations $\mathrm{s}^{43}$. Paired sera (from acute and convalescent phases) that exhibit positivation or increasing antibody titers in the second serum sample may be necessary to confirm virus diagnosis using $\mathrm{HI}$ or NT assays because these techniques do not discriminate IgG from IgM. In fact, the diagnosis of infections based exclusively on virus-specific serological testing cannot be used alone. However, these serologic techniques are useful for the diagnosis of travelers who return from geographic areas affected by CHIKV or MAYV. Moreover, cross-reactions could occur with antibodies produced in infections by other viruses of the Semliki forest serogroup ${ }^{20}$. Thus, considering that CHIKV and MAYV could circulate in the same region, it will be necessary to use techniques able to detect the viral genome (RT-PCR) or a NT that is highly species-specific to differentiate infections. Additionally, it will be necessary to develop better diagnostic tests that are simple, fast, specific and reliable for the diagnosis of CHIKV and MAYV.

\section{TREATMENT OF PATIENTS AND VACCINES TO PREVENT CHIKV AND MAYV INFECTIONS}

There are no specific drugs for routine use against CHIKV and MAYV. Patients are maintained in repose and are symptomatically treated with simple analgesics (acetaminophen, paracetamol) and/or nonsteroidal anti-inflammatory drugs (ibuprofen, naproxen), which can provide pain and fever relief. Corticosteroids have been used in a few cases of CHIKV but without proven efficacy ${ }^{43}$. Chloroquine, an anti-malaria drug, has been used to treat chronic arthritis caused by CHIKV with encouraging results ${ }^{44}$. Ribavirin (200mg twice per day for seven days) has been shown to be effective for relieving crippling lower limb pains and improved the resolution of these manifestations ${ }^{45}$. The combination of interferon alpha (IFN- $\alpha$ ) $2 \mathrm{~b}$ and ribavirin produces a synergistic anti-CHIKV effect, as demonstrated in an in vitro study ${ }^{46}$. Passive immunization could be an appropriate preventive and therapeutic option for Chikungunya and Mayaro fevers because no alternative therapy is available ${ }^{47}$. In a mouse model, human polyvalent immune globulins extracted and purified from plasma samples of donors in the convalescent phase of CHIKV infection exhibited high neutralizing activity, as well as a powerful therapeutic and prophylactic action against CHIKV infection ${ }^{48}$. Thus, passive immunization could be used for the treatment of Chikungunya fever and for prevention in patients at risk of severe CHIKV disease, such as adults with underlying conditions.

Some questions remain to be addressed to develop vaccines to protect against CHIKV and MAYV infections. What is the ideal animal model to test these vaccines? What is the better route for immunization? What is the cost of these vaccines? Considering that most outbreaks of CHIKV and MAYV occur in poor developing countries, it is important to create lowcost vaccines to immunize large populations. Some CHIKV vaccine candidates are currently under investigation. For example, a CHIKV particle-like vaccine has been investigated in a phase I clinical trial ${ }^{49}$. Recently, a vaccine candidate for MAYV that uses the internal ribosome entry site (IRES) of the encephalomyocarditis virus to replace the viral subgenomic promoter was able to change the host-specific mechanism of structural protein gene expression. This MAYV vaccine has been tested in immunocompetent mice and interferon receptor-knockout mice. In both animal models, the vaccine candidate was able to produce viral attenuation, the induction of neutralizing antibodies and efficacy against a lethal challenge with MAYV ${ }^{50}$. Further studies with this vaccine are ongoing. Finally, there is hope that vaccines to prevent infections by CHIKV and MAYV will be available in the near future.

\section{FINAL REMARKS}

CHIKV and MAYV result in relevant public health problems in the Americas, particularly Brazil. CHIKV, a virus transmitted by Aedes that causes large epidemics of febrile illness with joint involvement, has only recently been introduced to Brazil. The virus will likely spread throughout the country and become a national calamity similar to dengue. MAYV is phylogenetically related to CHIKV and causes outbreaks of febrile disease with articular involvement in the Amazon region. MAYV may emerge in other regions of the country if it adapts to Aedes transmission. There is little information regarding the febrile illnesses produced by these viruses. CHIKV and MAYV fevers can be confused with dengue fever. These facts, combined with the fact that a laboratory diagnosis of CHIKV and MAYV is only performed in a few places, could lead to the erroneous impression that outbreaks cause by CHIKV and MAYV are not present in populated regions of Brazil. 


\section{CONFLICT OF INTEREST}

The authors declare that there is no conflict of interest.

\section{FINANCIAL SUPPORT}

We gratefully acknowledge the National Council of Scientific and Technological Development [Conselho Nacional de Desenvolvimento Cientifico e Tecnológico (CNPq)], for the post-doctoral grant.

\section{REFERENCES}

1. Koonin E. On the origin of cells and viruses: primordial virus world scenario. Ann NY Acad Sci 2009; 1178:47-64.

2. Figueiredo LT. Emergent arboviruses in Brazil. Rev Soc Bras Med Trop 2007; 40:224-229.

3. Pybus OG, Rambaut A. Evolutionary analysis of the dynamics of viral infectious disease. Nat Rev Genet 2009; 10:540-550.

4. Hamer DH, Chen LH. Chikungunya: Establishing a New Home in the Western Hemisphere. Ann Intern Med 2014; [Cited 2014 Oct]. Available at: http://annals.org/article.aspx?articleid=1906848/.

5. Abad-Franch F, Grimmer GH, Paula VS, Figueiredo LT, Braga WS, Luz SL. Mayaro virus infection in amazonia: a multimodel inference approach to risk factor assessment. PLoS Negl Trop Dis 2012; 26:1846.

6. Weaver SC, Reisen WR. Present and Future Arboviral Threats. Antiviral Res 2010; 85:328.

7. Arias-Goeta C, Moutailler S, Mousson L, Zouache K, Thiberge JM, Caro V, et al. Chikungunya virus adaptation to a mosquito vector correlates with only few point mutations in the viral envelope glycoprotein. Infect Genet Evol 2014; 24:116-126.

8. Paul D, Bartenschlager R. Architecture and biogenesis of plusstrand RNA virus replication factories. World J Virol 2013; 2:32-48.

9. Khan AH1, Morita K, Parquet Md Mdel C, Hasebe F, Mathenge EG, Igarashi A. Complete nucleotide sequence of chikungunya virus and evidence for an internal polyadenylation site. J Gen Virol 2002; 83:3075-3084.

10. Khan M, Dhanwani R, Kumar JS, Rao PV, Parida M. Comparative evaluation of the diagnostic potential of recombinant envelope proteins and native cell culture purified viral antigens of Chikungunya virus. J Med Virol 2014; 86:1169-1175.

11. Jones KE, Patel NG, Levy MA, Storeygard D, Balk JL, et al. Global trends in emerging infectious diseases. Nature 2008; 451:990-994.

12. Pinheiro FP, Freitas RB, Rosa JFT, Gabbay YB, Mello WA, Le Duc JW. An outbreak of Mayaro virus disease in Belterra, Brazil. I. Clinical an virological findings. Am J Trop Med Hyg 1981; 30:674-681.

13. Mourão MPG, Mota BR, Souza GS, Bastos MS, Gimaque JBL, Galusso ES, et al. Mayaro fever in Manaus, Brazil, 2007-2008. Vector Borne Zoonotic Dis 2012; 12:42-46.

14. Robinsonm C. An epidemic of virus disease in Southern Province, Tanganyika Territory, in 1952-1953. I. Clinical features. Trans Royal Society Trop Med Hyg 1955, 49:28-32.

15. Schilte C, Couderc T, Chretien F, Sourisseau M, Gangneux N, Guivel-Benhassine F, et al. Type I IFN controls Chikungunya virus via its action on nonhematopoietic cells. J Exp Med 2010; 207: 429-442.
16. Tsetsarkin KA, Vanlandingham DL, McGee CE, Higgs S. A single mutation in chikungunya virus affects vector specificity and epidemic potential. PLoS Pathog 2007; 3:201.

17. Labadie K, Larcher T, Joubert C, Mannioui A, Delache B, Brochard P. Chikungunya disease in nonhuman primates involves long-term viral persistence in macrophages. J Clin Invest 2010; 120:894-906.

18. Kelvin AA, Banner D, Silvi G, Moro ML, Spataro N, Gaibani P, et al. Inflammatory cytokine expression is associated with Chikungunya virus resolution and symptom severity. PLoS Negl Trop Dis 2011; 5:1279.

19. Dupuis-Maguiraga L, Noret M, Brun S, Le Grand R, Gras G, Roques P. Chikungunya disease: infection-associated markers from the acute to the chronic phase of arbovirus-induced arthralgia. PLoS Negl Trop Dis 2012; 6:1446.

20. Caglioti C, Lalle E, Castilletti C, Carletti F, Capobianchi MR, Bordi L. Chikungunya virus infection: an overview. New Microbiol 2013; 36:211-227.

21. Simon F, Javelle E, Oliver M, Leparc-Goffart I, Marimoutou C. Chikungunya Virus Infection. Curr Infect Dis Rep 2011; 13:218228.

22. Kennedy AC, Fleming J, Solomon L. Chikungunya viral arthropathy: a clinical description. J Rheumatol 1980; 7:231-236.

23. Sam IC, Loong SK, Michael JC, Chua CL, Sulaiman WY, Vythillingam I, et al. Genotypic and phenotypic characterization of Chikungunya virus of different genotypes from Malaysia. PLoS One 2012; 7:50476.

24. Kalantri SP, Joshi R, Riley LW. Chikungunya epidemic: an Indian perspective. Natl Med J India 2006; 19:315-322.

25. Arias-Goeta C, Moutailler S, Mousson L, Zouache K, Thiberge JM, Caro V, et al. Chikungunya virus adaptation to a mosquito vector correlates with only few point mutations in the viral envelope glycoprotein. Infect Genet Evol 2014; 24:116-126.

26. World Health Organization. WHO report of outbreaks of Chikungunya 2005, 2006, 2007. WHO; 2007.

27. Center for Disease Control and Prevention (CDC). First Reports of Chikungunya in Western hemisphere. Press Release. CDC; 2007.

28. Fischer M, Staples JE, Arboviral Diseases Branch, National Center for Emerging and Zoonotic Infectious Diseases, Center for Disease Control and Prevention (CDC). Notes from the field: chikungunya virus spreads in the Americas - Caribbean and South America, 2013-2014. MMWR Morb Mortal Wkly Rep 2014; 63:500-501.

29. Vega-Rúa A, Zouache K, Girod R, Failloux A, Lourenço-deOliveira R. High vector competence of Aedes aegypti and Aedes albopictus from ten American countries as a crucial factor of the spread of Chikungunya. J Virol 2014; [Cited 2014 October]. Available at: http://jvi.asm.org/content/early/2014/03/20/JVI.0037014.short/.

30. Gallian P, Lamballerie X, Salez N, Piorkowski G, Richard P, Paturel L, et al. Prospective detection of chikungunya virus in blood donors, Caribbean 2014. Blood 2014; 123:3679-3681.

31. Pan American Health Organization (PAHO). Chikungunya outbreaks. PAHO; 2014.

32. Ministério da Saúde. Boletins sobre Chikungunya, 2001-2014. Brasília: Ministério da Saúde; 2014.

33. Anderson CR, Downs WG, Wattley GH, Ahin NH, Reese AA. Mayaro virus: a new human disease agent. II Isolation from blood of patients in Trinidad. Am J Trop Med Hyg 1957; 6:1012-1016.

34. Powers AM, Aguilar PV, Chandler LJ, Brault AC, Meakins TA, Watts D, et al. Genetic relationships among Mayaro and Una viruses suggest distinct patterns of transmission. Am J Trop Med Hyg 2006; 75:461-469. 
35. Figueiredo LTM, Nogueira RMR, Cavalcanti SMB, Rosa AT, Schatzmayr H. Study of two different enzyme immunoassays for the detection of Mayaro virus antibodies. Mem Inst Oswaldo Cruz 1989; 84:303-307.

36. Coimbra TL, Santos CL, Suzuki A, Petrella SM, Bisordi I, Nagamori $\mathrm{AH}$, et al. Mayaro virus: imported cases of human infection in São Paulo State, Brazil. Rev Inst Med Trop Sao Paulo 2007; 49:221-224.

37. Bronzoni RVM, Moreli ML, Cruz ACR, Figueiredo LTM. Multiplex-nested-PCR for Brazilian Alphavirus diagnosis. Trans R Soc Trop Med Hyg 2004; 98:456-461

38. Bronzoni RVM, Nogueira RMR, Nunes M, Figueiredo LTM. Detection and identification of Brazilian alphaviruses and flaviviruses by multiplex RT-PCR. J Clin Microbiol 2005; 43:696-702.

39. Figueiredo LT, Maciel MAV, Shope R. Identification of viruses, Togaviridae, Alphavirus: identification of Chikungunya virus from Central African Republic. Annual Report for 1986. Yale Arbovirus Research Unit-International Center for Arboviruses 1986; 1986:25-26.

40. Figueiredo LTM. Togaviridae-Alphavirus. In: Oliveira LHS, editor. Virologia Humana. Rio de Janeiro: Cultura Médica; 1994. p. 213222.

41. Greiser-Wilke I, Moennig V, Kaaden O, Figueiredo LTM. Most alphaviruses share a conserved epitopic region on their nucleocapsid protein. Journal General Virol 1989; 70:743-748.

42. Yap G, Pok KY, Lai YL, Hapuarachchi HC, Chow A, Leo YS, et al. Evaluation of Chikungunya diagnostic assays: differences in sensitivity of serology assays in two independent outbreaks. PLoS Negl Trop Dis 2010; 4:753.
43. Taubitz W, Cramer JP, Kapaun A, Pfeffer M, Drosten C, Dobler G, et al. Chikungunya fever in travelers: clinical presentation and course. Clin Infect Dis 2007; 45:1-4.

44. Brighton SW. Chloroquine phosphate treatment of chronic Chikungunya arthritis: an open pilot study. S Afr Med J 1984; 66:217-218

45. Ravichandran R, Manian M. Ribavirin therapy for Chikungunya arthritis. J Infect Dev Ctries 2008; 2:140-142.

46. Briolant S, Garin D, Scaramozzino N, Jouan A, Crance JM. In vitro inhibition of Chikungunya and Semliki Forest viruses replication by antiviral compounds: synergistic effect of interferon-alpha and ribavirin combination. Antiviral Res 2004; 61:111-117.

47. Dessain SK, Adekar SP, Berry JD. Exploring the native human antibody repertoire to create antiviral therapeutics. Curr Top Microbiol Immunol 2008; 317:155-183.

48. Couderc T, Khandoudi N, Grandadam M, Visse C, Gangneux N, Bagot S, et al. Prophylaxis and therapy for Chikungunya virus infection. J Infect Dis 2009; 200:516-523.

49. Chang LJ, Dowd KA, Mendoza FH, Saunders JG, Sitar S, Plummer SH, et al. The VRC 311 Study Team. Safety and tolerability of chikungunya virus-like particle vaccine in healthy adults: a phase 1 dose-escalation trial. Lancet 2014; [Cited 2014 October]. Available at: http://www. thelancet.com/journals/lancet/article/PIIS0140-6736(14)61185-5/ abstract/.

50. Weise WJ, Hermance ME, Forrester N, Adams AP, Langsjoen R, Gorchakov R, et al. A novel live-attenuated vaccine candidate for mayaro Fever. PLoS Negl Trop Dis 2014; 8:2969. 